PROCEEDINGS OF THE

AMERICAN MATHEMATICAL SOCIETY

Volume 128, Number 6, Pages 1673-1683

S 0002-9939(00)05493-9

Article electronically published on February 7, 2000

\title{
EXISTENCE AND UNIQUENESS THEOREMS FOR SINGULAR ANISOTROPIC QUASILINEAR ELLIPTIC BOUNDARY VALUE PROBLEMS
}

\author{
S. HILL, K. S. MOORE, AND W. REICHEL
}

(Communicated by Lesley M. Sibner)

\begin{abstract}
On bounded domains $\Omega \subset \mathbb{R}^{2}$ we consider the anisotropic problems $u^{-a} u_{x x}+u^{-b} u_{y y}=p(x, y)$ in $\Omega$ with $a, b>1$ and $u=\infty$ on $\partial \Omega$ and $u^{c} u_{x x}+u^{d} u_{y y}+q(x, y)=0$ in $\Omega$ with $c, d \geq 0$ and $u=0$ on $\partial \Omega$. Moreover, we generalize these boundary value problems to space-dimensions $n>2$. Under geometric conditions on $\Omega$ and monotonicity assumption on $0<p, q \in \mathcal{C}^{\alpha}(\bar{\Omega})$ we prove existence and uniqueness of positive solutions.
\end{abstract}

\section{IntRoduCtion}

In [6], the first two authors proved that if $\Omega \subset \mathbb{R}^{2}$ is a smooth, bounded, convex domain and $p$ is continuous and positive on $\bar{\Omega}$, then there exists a positive solution $u \in \mathcal{C}^{2}(\Omega)$ to

$$
\begin{aligned}
u^{-a} u_{x x}+u^{-b} u_{y y}-p(x, y) & =0 \text { in } \Omega \\
u & =\infty \text { on } \partial \Omega
\end{aligned}
$$

where $a \geq b>1$ and the boundary condition is understood in the sense that $u(x, y) \rightarrow \infty$ whenever $(x, y) \in \Omega$ tends to a point $\left(x_{0}, y_{0}\right)$ on $\partial \Omega$. In the case $a=b>1$, problem (11) is isotropic and represents a special case of the equation $\Delta u+f(x, u)=0$ in $\Omega$ with infinite boundary conditions. Much research (including existence, uniqueness and asymptotic behavior) has been done on the isotropic case, starting with the work of Keller [7] and Osserman [12], where a large class of fast-growing nonlinearities $f$ was studied. For an overview of subsequent work on the isotropic boundary blow-up problem see Bandle, Marcus 1], 2] and Lazer, McKenna [9, 10].

In this paper we come back to the anisotropic problem and show that a similar existence result holds in $\mathbb{R}^{n}$. Moreover, we will show that the positive solution is unique. Specifically, if $\Omega \subset \mathbb{R}^{n}$ is a smooth, bounded domain which satisfies a weighted star-shapedness condition (a requirement which we will define in Section

Received by the editors July 9, 1998.

2000 Mathematics Subject Classification. Primary 35J65; Secondary 35J70.

Key words and phrases. Anisotropic singular equations, comparison principles.

(C)2000 American Mathematical Society 
2), under certain conditions on $p$, there exists a unique positive solution to

$$
\begin{aligned}
\sum_{j=1}^{n} u^{-d_{j}} u_{x_{j} x_{j}}-p(x) & =0 \text { in } \Omega, \\
u & =\infty \text { on } \partial \Omega,
\end{aligned}
$$

where $d_{1}, \ldots \geq d_{n}>1$. The main difficulty in the treatment of (2) is the lack of the usual comparison principle between upper and lower solution due to the anisotropic character of the equation. However, in Section 2 we will state comparison principles which are suitable for (2).

Likewise, our methods apply to prove existence and uniqueness of positive solutions to the problem

$$
\begin{aligned}
\sum_{j=1}^{n} u^{e_{j}} u_{x_{j} x_{j}}+q(x) & =0 \text { in } \Omega, \\
u & =0 \text { on } \partial \Omega,
\end{aligned}
$$

where $e_{1}, \ldots \geq e_{n} \geq 0$. The existence part for problem (13) on convex domains was treated by Choi, Lazer, McKenna [3] and Choi, McKenna [4], where uniqueness was left as an open problem. Partial results towards uniqueness in dimension $n=2$ were given by Lair, Shaker [8] and Reichel [13]. For non-convex domains, very little is known, cf. 4]. By our method we can show existence and uniqueness for a class of functions $q$ and for a class of domains which satisfy a weighted star-shapedness condition (cf. Section 2). This class of domains contains non-convex domains, but it does not contain all convex domains; cf. Section 2 Therefore, our existence results complement those in 4], whereas the uniqueness part is new.

We point out that in many aspects (2) and (3) can be treated by very similar methods. This was already observed by Lazer, McKenna in [10].

Our paper is structured as follows: In Section 2 we prove two comparison lemmas and in Section 3 we use these lemmas to prove existence and uniqueness of a solution to (2). In Section 4 we show how our method can be applied to (3). In the final section we pose some open problems.

\section{Comparison lemmas}

One of our main tools is the following comparison lemma. We state it in a more general form than we need, since it may be useful for other applications. A closely related version was found by Choi, Lazer and McKenna in [3].

Lemma 2.1. Consider the problem

$$
\begin{aligned}
G(u):=\sum_{j=1}^{n} g_{j}(x, u) u_{x_{j} x_{j}}+g_{n+1}(x, u) & =0 \text { in } \Omega, \\
u & =h \text { on } \partial \Omega,
\end{aligned}
$$

where the functions $g_{i}=g_{i}(x, s)$ defined on $\Omega \times(0, \infty)$ are nonincreasing in $s>0$ for $i=1, \ldots, n+1$ and nonnegative for $i=1, \ldots, n$. Suppose there exists $\phi, \psi \in$ $\mathcal{C}(\bar{\Omega}) \cap \mathcal{C}^{2}(\Omega)$ with $\phi, \psi>0, \phi_{x_{1} x_{1}}, \psi_{x_{1} x_{1}}, \ldots, \phi_{x_{n} x_{n}}, \psi_{x_{n} x_{n}} \geq 0$ in $\Omega$. Also, suppose $G(\phi)<0, G(\psi)>0$ and $\left.\phi\right|_{\partial \Omega} \geq h \geq\left.\psi\right|_{\partial \Omega}$. If $u \in \mathcal{C}(\bar{\Omega}) \cap \mathcal{C}^{2}(\Omega)$ is a positive solution to (4), then $\phi \geq u \geq \psi$. 
Remark. 1) In the case that $g_{1}, \ldots, g_{n}$ are positive functions of $x$ only, this lemma amounts to the standard comparison theorem for linear uniformly elliptic operators with a nonincreasing nonlinearity $g_{n+1}$, and it even holds without the assumptions on $\phi_{x_{i} x_{i}}, \psi_{x_{i} x_{i}}$. Lemma 2.1 can be viewed as an extension of the standard comparison principle to a class of quasilinear operators, which includes, e.g. (2).

2) The condition $\left.\phi\right|_{\partial \Omega} \geq h$ is used to ensure that a negative infimum of $\phi-u$ cannot be attained on $\partial \Omega$. If $\lim \phi(x)=\infty$ uniformly in $x$ as $x \rightarrow \partial \Omega$, then any negative infimum of $\phi-u$ is attained in $\Omega$ and the conclusion of Lemma 2.1 still holds for such boundary values of $\phi$.

Proof. Let $u>0$ solve (4) and suppose that the above inequality does not hold; i.e., suppose there exists an $x \in \Omega$ such that $(\phi-u)(x)<0$. Let $x_{0} \in \Omega$ be the point where the infimum of $\phi-u$ is attained. Then $(\phi-u)_{x_{j} x_{j}}\left(x_{0}\right) \geq 0$ for $j=1, \ldots, n$ and since $G(u)=0$, we have at $x_{0}$

$$
\begin{aligned}
G(\phi)= & G(\phi)-G(u) \\
= & \sum_{j=1}^{n} g_{j}\left(x_{0}, \phi\right) \phi_{x_{j} x_{j}}+g_{n+1}\left(x_{0}, \phi\right)-\sum_{j=1}^{n} g_{j}\left(x_{0}, u\right) u_{x_{j} x_{j}}-g_{n+1}\left(x_{0}, u\right) \\
= & \sum_{j=1}^{n}\left(g_{j}\left(x_{0}, \phi\right)-g_{j}\left(x_{0}, u\right)\right) \phi_{x_{j} x_{j}}+\sum_{j=1}^{n} g_{j}\left(x_{0}, u\right)(\phi-u)_{x_{j} x_{j}} \\
& +g_{n+1}\left(x_{0}, \phi\right)-g_{n+1}\left(x_{0}, u\right) \geq 0 .
\end{aligned}
$$

But $G(\phi)<0$. Therefore, $u \leq \phi$ in $\Omega$. Similarly, $\psi \leq u$ in $\Omega$.

The following geometric notion is a technical condition that is used for our uniqueness proof.

Definition. For a set of exponents $f_{1}, f_{2}, \ldots, f_{n}>0$, a bounded domain $\Omega \subset \mathbb{R}^{n}$ which contains the origin satisfies a WEIGHTED STAR-SHAPEDNESS CONDITION if $\left(\lambda^{f_{1}} x_{1}, \ldots, \lambda^{f_{n}} x_{n}\right) \in \Omega$ for all $\left(x_{1}, \ldots, x_{n}\right) \in \Omega$ and $\lambda \in(0,1)$.

Notice that for $f_{1}=\ldots=f_{n}$, the weighted star-shapedness condition is the usual star-shapedness condition with respect to the origin. In general, weighted star-shapedness and star-shapedness are not comparable. In the example in Figure 1 the $(1,2)$-weighted star-shapedness means that $\left(\lambda x, \lambda^{2} y\right)$ lies in $\Omega$ for $(x, y) \in \Omega$ and $0<\lambda<1$, i.e., the piece of parabola between the vertex $(0,0)$ and the point $(x, y)$ has to lie in $\Omega$.

The next condition identifies a large class of domains that satisfy the weighted star-shapendess condition for any set of positive exponents. In dimension $n=2$ this condition was introduced by Reichel [13] as the interior rectangle condition (see Figure 2).

Definition. A bounded domain $\Omega \subset \mathbb{R}^{n}$ which contains the origin satisfies the INTERIOR BOX CONDITION if for all $\left(x_{1}, \ldots, x_{n}\right) \in \partial \Omega$ the box $\left[0, x_{1}\right) \times \ldots \times\left[0, x_{n}\right)$ lies in $\Omega$.

The interior box condition is less general than star-shapedness with respect to 0 . But the interior box condition implies the weighted star-shapedness condition for any set of positive exponents. We introduce the interior box condition because it is easier to verify than the weighted star-shapedness condition. Notice that both the weighted star-shapedness condition and the interior box condition are not invariant 


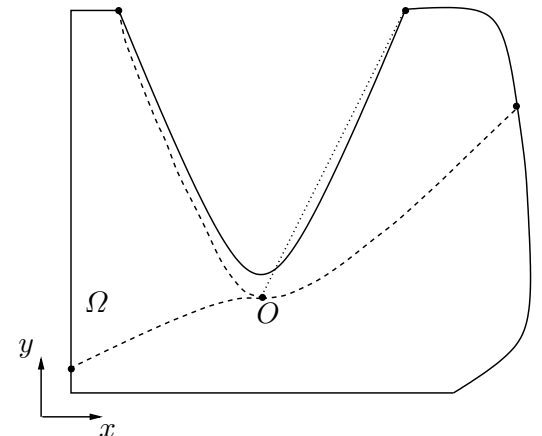

(1,2)-weighted starshaped not starshaped w.r.t. $O$

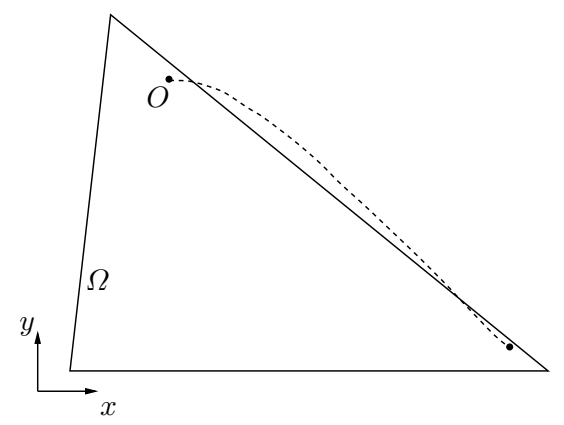

not $(1,2)$-weighted starshaped convex

Figure 1. Weighted star-shapedness

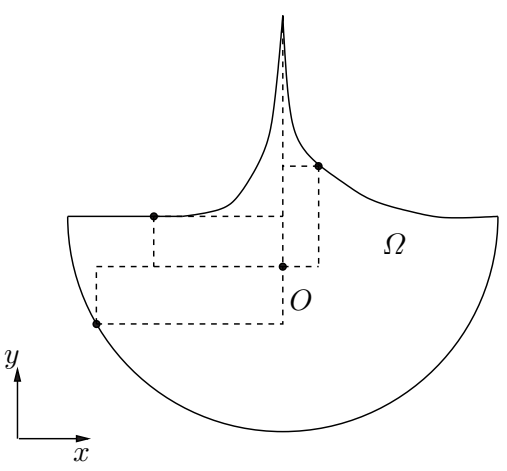

interior box condition, non-convex

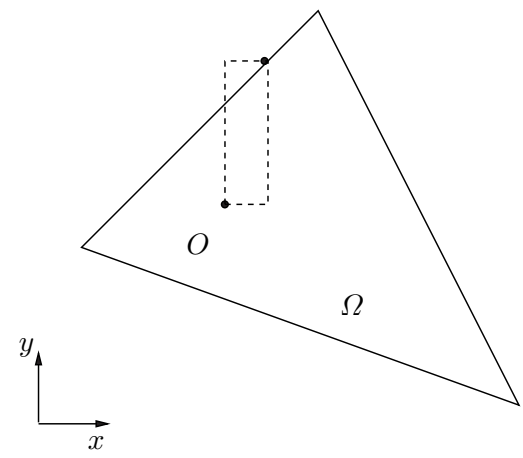

convex, no interior box condition

Figure 2. Interior box condition

under rotations, which reflects the fact that the differential equation (2) is also not invariant under rotations.

The following comparison lemma is one of the main tools for our existence and uniqueness result. Compared to Lemma 2.1 it requires no sign-condition for the second-derivatives of a sub-, supersolution pair. Instead, it requires weighted starshapedness of the underlying domain and a corresponding monotonicity assumption on the zero-order term.

Lemma 2.2. For $u \in \mathcal{C}^{2}(\Omega)$ we define

$$
F(u):=\sum_{j=1}^{n} u^{-d_{j}} u_{x_{j} x_{j}}-p(x),
$$

where $d_{1}, \ldots, d_{n}>1$, and we suppose that $\Omega \subset \mathbb{R}^{n}$ satisfies the weighted starshapedness condition for the exponents $\left(d_{1}-1\right) / 2, \ldots,\left(d_{n}-1\right) / 2$. For $\lambda \in(0,1]$ and $x=\left(x_{1}, \ldots, x_{n}\right) \in \Omega$, define

$$
x_{\lambda}:=\left(\lambda^{\frac{d_{1}-1}{2}} x_{1}, \lambda^{\frac{d_{2}-1}{2}} x_{2}, \ldots, \lambda^{\frac{d_{n}-1}{2}} x_{n}\right) .
$$


Let $p \in \mathcal{C}(\bar{\Omega})$ be a positive function such that

$$
p\left(x_{\lambda}\right) \geq p(x) \text { for all } x \in \Omega \text { and all } \lambda \in(0,1] .
$$

Let $v, w \in \mathcal{C}^{2}(\Omega) \cap \mathcal{C}(\bar{\Omega})$ be positive in $\Omega$ and suppose they satisfy $F(v) \leq 0$ and $F(w) \geq 0$ in $\Omega$. If $\inf _{x \in \partial \Omega} v \geq \max _{\Omega^{\prime} \subset \subset \Omega} w$ for every compact subset $\Omega^{\prime}$ of $\Omega$, then $v \geq w$ in $\Omega$.

Proof. Due to the weighted star-shapedness condition we can define $w_{\lambda}(x)=\lambda w\left(x_{\lambda}\right)$ for $\lambda \in(0,1), x=\left(x_{1}, \ldots, x_{n}\right) \in \Omega$. Note that $\left(w_{\lambda}\right)_{x_{j} x_{j}}(x)=\lambda^{d_{j}} w_{x_{j} x_{j}}\left(x_{\lambda}\right)$ and therefore, since $F(w) \geq 0$ on $\Omega$, we have

$$
\begin{aligned}
F\left(w_{\lambda}(x)\right) & =\sum_{j=1}^{n} w^{-d_{j}}\left(x_{\lambda}\right) w_{x_{j} x_{j}}\left(x_{\lambda}\right)-p(x) \\
& =F\left(w\left(x_{\lambda}\right)\right)+p\left(x_{\lambda}\right)-p(x) \geq 0 .
\end{aligned}
$$

Now for $t \in[0,1]$, define

$$
w_{\lambda, t}(x):=t v(x)+(1-t) w_{\lambda}(x)
$$

and for $j=1, \ldots, n$ consider

$$
\mu_{j}:=\int_{0}^{1} \frac{d}{d t}\left(w_{\lambda, t}^{-d_{j}}\left(w_{\lambda, t}\right)_{x_{j} x_{j}}\right) d t .
$$

Then we have

$$
\mu_{j}=\left.w_{\lambda, t}^{-d_{j}}\left(w_{\lambda, t}\right)_{x_{j} x_{j}}\right|_{0} ^{1}=v^{-d_{j}} v_{x_{j} x_{j}}-w_{\lambda}^{-d_{j}}\left(w_{\lambda}\right)_{x_{j} x_{j}} .
$$

Also, differentiating inside the integral in (8)), we see that

$$
\begin{aligned}
\mu_{j} & =\left(v-w_{\lambda}\right)_{x_{j} x_{j}} \int_{0}^{1} w_{\lambda, t}^{-d_{j}} d t-d_{j}\left(v-w_{\lambda}\right) \int_{0}^{1}\left(w_{\lambda, t}\right)_{x_{j} x_{j}} w_{\lambda, t}^{-d_{j}-1} d t \\
& =a_{j j}\left(v-w_{\lambda}\right)_{x_{j} x_{j}}+c_{j}\left(v-w_{\lambda}\right),
\end{aligned}
$$

where $a_{j j}:=\int_{0}^{1} w_{\lambda, t}^{-d_{j}} d t>0$ and $c_{j}:=-d_{j} \int_{0}^{1}\left(w_{\lambda, t}\right)_{x_{j} x_{j}} w_{\lambda, t}^{-d_{j}-1} d t$. From the above expression for $\mu_{j}$ and (9), we see that

$$
\begin{aligned}
L(v-w) & :=\sum_{j=1}^{n} a_{j j}\left(v-w_{\lambda}\right)_{x_{j} x_{j}}+c_{j}\left(v-w_{\lambda}\right) \\
& =\sum_{j=1}^{n} \mu_{j}=F(v)-F\left(w_{\lambda}\right) \leq 0
\end{aligned}
$$

since $F(v) \leq 0$ and $F\left(w_{\lambda}\right) \geq 0$. Notice that, even in the case where $v$ is unbounded, on compact subsets of $\Omega$ we find that the operator $L$ is uniformly elliptic with bounded coefficients.

Now define $S=\left\{\lambda \in(0,1): w_{\lambda} \leq v\right\}$. Since $w_{\lambda}$ tends to 0 uniformly in $\bar{\Omega}$ as $\lambda \rightarrow 0$, and since $\min _{\bar{\Omega}} v>0$, we note that $S \neq \emptyset$. Let $\lambda^{*}=\sup _{\lambda \in S} \lambda$. Note that if $\lambda^{*}=1$, we have $w=w_{1} \leq v$ and the proof of the lemma is complete. We claim that $\lambda^{*}=1$. To see this, suppose $\lambda^{*}<1$. By the maximality of $\lambda^{*}$ there exists a "touching point" $x_{0} \in \bar{\Omega}$ with $w_{\lambda^{*}}\left(x_{0}\right)=v\left(x_{0}\right)$. By the hypotheses, inf $\partial \Omega v>$ $\max _{\bar{\Omega}} w_{\lambda^{*}}$. Therefore $x_{0}$ lies in $\Omega$. Since $L\left(v-w_{\lambda^{*}}\right) \leq 0$ and $v \geq w_{\lambda^{*}}$, the strong minimum principle implies $v \equiv w_{\lambda^{*}}$. But this contradicts $\inf _{\partial \Omega} v>\max _{\bar{\Omega}} w_{\lambda^{*}}$. Therefore, $\lambda^{*}=1$, and the proof is complete. 


\section{Existence AND UNiqueness FOR (2)}

Theorem 3.1. Let $\Omega \subset \mathbb{R}^{n}$ be a smooth, bounded domain which contains the origin and which satisfies the weighted star-shapedness condition with exponents $\left(d_{1}-1\right) / 2, \ldots,\left(d_{n}-1\right) / 2>0$ and let $p \in \mathcal{C}^{\alpha}(\bar{\Omega})$ be a positive function that satisfies the monotonicity assumption (7). Then there exists a unique positive solution $u \in \mathcal{C}^{2}(\Omega)$ to the problem

$$
\begin{aligned}
\sum_{j=1}^{n} u^{-d_{j}} u_{x_{j} x_{j}}-p(x) & =0 \text { in } \Omega \\
u & =\infty \text { on } \partial \Omega .
\end{aligned}
$$

Proof. Without loss of generality we may assume $d_{n}=\min \left\{d_{j}: j=1, \ldots, n\right\}$. The hypotheses on $p$ imply the existence of constants $M_{1}, M_{2}>0$ such that $M_{1} \leq$ $p(x) \leq M_{2}$ for all $x \in \bar{\Omega}$. For fixed $m>0$ let $\psi_{m}$ be the solution of

$$
\begin{aligned}
& \psi^{\prime \prime}-\left(M_{2}+1\right) \psi^{d_{n}}=0 \text { in }(-L, L), \\
& \psi(-L)=\psi(L)=m,
\end{aligned}
$$

where $2 L=\operatorname{diam} \Omega$. Notice that the solution $\psi_{m}$ is uniquely determined and that $\psi_{m} \geq 2 \epsilon$ in $(-L, L)$ for some $\epsilon>0$ independent on $m$. From now on we consider $\psi_{m}(x)=\psi_{m}\left(x_{n}\right)$ as a function on the strip $S=\left\{x \in \mathbb{R}^{n}:-L<x_{n}<L\right\}$, which satisfies $\left(\psi_{m}\right)_{x_{n} x_{n}}-\left(M_{2}+1\right) \psi_{m}^{d_{n}}=0$ in $S$. Modulo a translation of the strip $S$ in the $x_{n}$-direction we may assume that $\Omega$ is contained in $S$.

Next we apply a standard cut-off procedure, i.e., above the constant $m$ and below the function $\psi_{m}$ we cut off the nonlinear terms in (10) in a smooth way. For $j=1, \ldots, n$ and $x \in \Omega$, define

$$
f_{m}^{(j)}(x, u)= \begin{cases}\left(\psi_{m}(x)-\epsilon\right)^{-d_{j}} & \text { if } 0<u \leq \psi_{m}(x)-\epsilon \\ u^{-d_{j}} & \text { if } \psi_{m}(x) \leq u \leq m \\ (m+1)^{-d_{j}} & \text { if } m+1 \leq u\end{cases}
$$

and extend $f_{m}^{(j)}$ in a nonincreasing, smooth way in the strips $m \leq u \leq m+1$ and $\psi_{m}(x)-\epsilon \leq u \leq \psi_{m}(x)$. With this construction $f_{m}^{(j)}$ is a bounded, nonincreasing, $\mathcal{C}^{1}$-function that coincides with $u^{-d_{j}}$ in the region where $\psi_{m} \leq u \leq m$. Consider the problem

$$
\begin{aligned}
F_{m}(u):=\sum_{j=1}^{n} f_{m}^{(j)}(x, u) u_{x_{j} x_{j}}-p(x) & =0 \text { in } \Omega, \\
u & =m \text { on } \partial \Omega .
\end{aligned}
$$

Since (13) is a uniformly elliptic operator with bounded coefficients, the existence of a positive $\mathcal{C}^{2, \alpha}(\bar{\Omega})$-solution $u_{m}$ to (13) follows from Theorem 15.12 in Gilbarg, Trudinger [5]. Since $\psi_{m} \leq m$ we find that

$$
\begin{aligned}
F_{m}\left(\psi_{m}\right) & =f_{m}^{(n)}\left(x, \psi_{m}(x)\right)\left(\psi_{m}\right)_{x_{n} x_{n}}-p(x) \\
& =M_{2}+1-p(x)>0
\end{aligned}
$$

by (11), (12) and the restriction on $p$. Therefore, $\psi_{m}$ is a lower solution to (13). Likewise, if we define $\phi_{m}(x)=m$, then clearly

$$
F_{m}\left(\phi_{m}\right)=-p(x)<0
$$

and therefore $\phi_{m}$ is an upper solution to (13). Since $\left(\psi_{m}\right)_{x_{j} x_{j}},\left(\phi_{m}\right)_{x_{j} x_{j}} \geq 0$ for $j=1, \ldots, n$, the Comparison Lemma 2.1] applies and shows that the solution $u_{m}$ to 
(13) satisfies $\psi_{m} \leq u_{m} \leq \phi_{m}=m$. Therefore we can remove the cut-off and we find that $u_{m}$ solves

$$
\begin{aligned}
F(u):=\sum_{j=1}^{n} u^{-d_{j}} u_{x_{j} x_{j}}-p(x) & =0 \text { in } \Omega, \\
u & =m \text { on } \partial \Omega .
\end{aligned}
$$

Next we show that the sequence $u_{m}$ is uniformly bounded above on every compact subset $\Omega^{\prime}$ of $\Omega$. Since $\Omega^{\prime}$ can be covered by a finite number of balls $B_{R}(\tilde{x}) \subset \Omega$ with sufficiently small radius $R$, it is enough to establish an upper bound on such balls. Let $\Phi:[0, R) \rightarrow \mathbb{R}^{+}$solve

$$
\begin{aligned}
& \Phi^{\prime \prime}=\frac{M_{1}}{n} \Phi^{d_{n}} \text { in }[0, R), \\
& \Phi(R)=\infty, \quad \Phi^{\prime}(0)=0 .
\end{aligned}
$$

Note that we can assume $R$ to be so small that $\Phi \geq 1$ on $[0, R)$. On the ball $B_{R}(\tilde{x})$ the function $\phi(x)=\Phi(r)$ with $r=|x-\tilde{x}|$ is a $\mathcal{C}^{2}$-function. Since $\Phi^{\prime}(0)=0$, we find by the mean value theorem a point $\rho=\rho(r) \in(0, r)$ such that

$$
\frac{n-1}{r} \Phi^{\prime}(r)=(n-1) \Phi^{\prime \prime}(\rho) \leq(n-1) \Phi^{\prime \prime}(r),
$$

where the last estimate follows from (16). Therefore

$$
\Delta \phi=\Phi^{\prime \prime}+\frac{n-1}{r} \Phi^{\prime} \leq n \Phi^{\prime \prime} \leq p(x) \phi^{d_{n}}
$$

on the ball $B_{R}(\tilde{x})$. Moreover, $\partial_{x_{i}} \Phi(r)=\Phi^{\prime} \frac{\left(x_{i}-\tilde{x}_{i}\right)}{r}$, and by using that $\Phi$ is increasing and convex we find $\partial_{x_{i}}^{2} \Phi(r)=\Phi^{\prime}\left(\frac{1}{r}-\frac{\left(x_{i}-\tilde{x}_{i}\right)^{2}}{r^{3}}\right)+\Phi^{\prime \prime} \frac{\left(x_{i}-\tilde{x}_{i}\right)^{2}}{r^{2}} \geq 0$. Finally, this implies

$$
\sum_{i=1}^{n} \phi^{-d_{i}} \phi_{x_{i} x_{i}} \leq \phi^{-d_{n}} \sum_{i=1}^{n} \phi_{x_{i} x_{i}}=\phi^{-d_{n}} \Delta \phi \leq p(x)
$$

in $B_{R}(\tilde{x})$. Since $\phi(x) \rightarrow \infty$ uniformly as $x \rightarrow \partial B_{R}(\tilde{x})$, we can apply Lemma 2.1 to $u_{m}$ and $\phi$ on the ball $B_{R}(\tilde{x})$ and we find $u_{m} \leq \phi$ in $B_{R}(\tilde{x})$. If we choose $R^{\prime}<R$, then $u_{m}$ is bounded above uniformly in $m$ on any ball $B_{R^{\prime}}(\tilde{x}) \subset \Omega$. A covering argument implies a uniform $L^{\infty}$-bound on $u_{m}$ on each compact subset $\Omega^{\prime}$ of $\Omega$.

Since $u_{m}$ is also bounded away from zero by $u_{m} \geq \psi_{m} \geq 2 \epsilon$, we find on compact subsets $\Omega^{\prime} \subset \subset \Omega$ that the ellipticity coefficients of (15) in the range of $u_{m}$ are bounded and bounded away from zero uniformly with respect to $m$. Therefore we may apply the fundamental Hölder-estimates of Krylov, Safonov (cf. Gilbarg, Trudinger [5], Corollary 9.24) and find that $\left\|u_{m}\right\|_{\mathcal{C}^{\alpha}\left(\Omega^{\prime}\right)} \leq$ const. $\left(\Omega^{\prime}\right)$, where $\alpha=$ $\alpha\left(\Omega^{\prime}\right) \in(0,1)$. With this preliminary smoothness of the coefficients of (15) and the local boundedness of $u_{m}$ we can apply the standard interior Schauder-estimates and get for every compact subset $\Omega^{\prime}$ of $\Omega$ that $\left\|u_{m}\right\|_{\mathcal{C}^{2, \alpha}\left(\Omega^{\prime}\right)} \leq$ const. $\left(\Omega^{\prime}\right)$, where $\alpha=\alpha\left(\Omega^{\prime}\right)$. Hence on every compact subset we can extract a $\mathcal{C}^{2, \alpha^{\prime}}$-convergent subsequence, and with a final diagonalization argument we find that a subsequence of the $u_{m}$ converges for $m \rightarrow \infty$ in $\mathcal{C}_{\text {loc }}^{2}(\Omega)$ to a limit function $u \in \mathcal{C}^{2}(\Omega)$, which satisfies the differential equation in (10). To see that $u$ satisfies the boundary condition in (10), note that by Lemma 2.2 we have $u_{m} \leq u_{m+1}$ and therefore 
$u_{m}(x) \leq u(x)$ for all $x \in \Omega$. Letting $x \rightarrow \partial \Omega$, we have

$$
m=\lim _{x \rightarrow \partial \Omega} u_{m}(x) \leq \lim _{x \rightarrow \partial \Omega} u(x) .
$$

This holds for all $m$; therefore $u$ satisfies the boundary condition in (10).

To see that the solution is unique, suppose $u_{1}$ and $u_{2}$ both solve (10). The comparison Lemma 2.2 applies and shows $u_{1} \geq u_{2}$, if we notice that $\inf _{\partial \Omega} u_{1}=$ $\infty>\max _{\Omega^{\prime} \subset \subset \Omega} u_{2}$ holds. Repeating the argument with the roles of $u_{1}$ and $u_{2}$ reversed, we find $u_{1}=u_{2}$.

\section{Existence And uniqueness for (3)}

We formulate the necessary steps for problem (3), and we indicate where the proofs differ from the ones in Sections 2 and 3. The next lemma is yet another extension of the standard, linear comparison principle to a class of quasilinear operators including (3).

Lemma 4.1. Consider the problem

$$
\begin{aligned}
\mathcal{G}(u):=\sum_{j=1}^{n} g_{j}(x, u) u_{x_{j} x_{j}}+g_{n+1}(x, u) & =0 \text { in } \Omega, \\
u & =h \text { on } \partial \Omega,
\end{aligned}
$$

where the functions $g_{i}=g_{i}(x, s)$ defined on $\Omega \times(0, \infty)$ are nonnegative and nondecreasing in $s>0$ for $i=1, \ldots, n$ and $g_{n+1}=g_{n+1}(x, s)$ defined on $\Omega \times(0, \infty)$ is nonincreasing in $s>0$. Suppose there exists $\phi, \psi \in \mathcal{C}(\bar{\Omega}) \cap \mathcal{C}^{2}(\Omega)$ with $\phi, \psi>0$, $\phi_{x_{1} x_{1}}, \psi_{x_{1} x_{1}}, \ldots, \phi_{x_{n} x_{n}}, \psi_{x_{n} x_{n}} \leq 0$ in $\Omega$. Also, suppose $\mathcal{G}(\phi)<0, \mathcal{G}(\psi)>0$ and $\left.\phi\right|_{\partial \Omega} \geq h \geq\left.\psi\right|_{\partial \Omega}$. If $u \in \mathcal{C}(\bar{\Omega}) \cap \mathcal{C}^{2}(\Omega)$ is a positive solution to (17), then $\phi \geq u \geq \psi$.

Lemma 4.2. For $u \in \mathcal{C}^{2}(\Omega)$ we define

$$
\mathcal{F}(u):=\sum_{j=1}^{n} u^{e_{j}} u_{x_{j} x_{j}}+q(x),
$$

where $e_{1}, \ldots, e_{n} \geq 0$, and we suppose that $\Omega \subset \mathbb{R}^{n}$ satisfies the weighted starshapedness condition for the exponents $\left(e_{1}+1\right) / 2, \ldots,\left(e_{n}+1\right) / 2$. For $\lambda \in(0,1]$ and $x=\left(x_{1}, \ldots, x_{n}\right) \in \Omega$, define

$$
x_{\lambda}:=\left(\lambda^{\frac{e_{1}+1}{2}} x_{1}, \lambda^{\frac{e_{2}+1}{2}} x_{2}, \ldots, \lambda^{\frac{e_{n}+1}{2}} x_{n}\right) .
$$

Let $q \in \mathcal{C}(\bar{\Omega})$ be a positive function such that

$$
q\left(x_{\lambda}\right) \geq q(x) \text { for all } x \in \Omega \text { and all } \lambda \in(0,1] .
$$

Let $v, w \in \mathcal{C}^{2}(\Omega) \cap \mathcal{C}(\bar{\Omega})$ be positive in $\Omega$ and suppose they satisfy $\mathcal{F}(v) \leq 0$ and $\mathcal{F}(w) \geq 0$ in $\Omega$. If $\min _{\Omega^{\prime} \subset \subset \Omega} v \geq \max _{\partial \Omega} w$ for every compact subset $\Omega^{\prime}$ of $\Omega$, then $v \geq w$ in $\Omega$.

Proof. Let $v_{\lambda}(x)=\lambda^{-1} v\left(x_{\lambda}\right)$ for $\lambda \leq 1$ and $x \in \Omega$. Then $\left(\mathcal{F}\left(v_{\lambda}\right)\right)(x)=(\mathcal{F}(v))\left(x_{\lambda}\right)-$ $q\left(x_{\lambda}\right)+q(x) \leq 0$. Since $v_{\lambda} \rightarrow+\infty$ uniformly in $\bar{\Omega}$ as $\lambda \rightarrow 0$, we have $v_{\lambda} \geqq w$ in $\bar{\Omega}$ for small $\lambda$. At the maximal value $\lambda^{*}$ we have a touching point $x_{0} \in \bar{\Omega}$ with $v_{\lambda^{*}}\left(x_{0}\right)=w\left(x_{0}\right)$. By the hypotheses of the lemma we have $\min _{\bar{\Omega}} v_{\lambda^{*}}>\max _{\partial \Omega} w$, provided $\lambda^{*}<1$. Therefore $x_{0}$ lies in $\Omega$. Since $v_{\lambda^{*}}-w \geq 0$ satisfies an elliptic differential inequality, which is uniformly elliptic in compact subsets of $\Omega$, the strong minimum principle implies $v_{\lambda^{*}} \equiv w$. But this is impossible for $\lambda^{*}<1$. 
Theorem 4.3. Let $\Omega \subset \mathbb{R}^{n}$ be a smooth, bounded domain which contains the origin and which satisfies the weighted star-shapedness condition with exponents $\left(e_{1}+1\right) / 2, \ldots,\left(e_{n}+1\right) / 2>0$ and let $q \in \mathcal{C}^{\alpha}(\bar{\Omega})$ be a positive function that satisfies the monotonicity assumption (20). Then there exists a unique positive solution $u \in \mathcal{C}^{2}(\Omega) \cap \mathcal{C}(\bar{\Omega})$ to the problem

$$
\begin{aligned}
\sum_{j=1}^{n} u^{e_{j}} u_{x_{j} x_{j}}+q(x) & =0 \text { in } \Omega, \\
u & =0 \text { on } \partial \Omega .
\end{aligned}
$$

Proof. We assume $e_{n}=\min \left\{e_{j}: j=1, \ldots, n\right\}$ and $0<M_{1} \leq q(x) \leq M_{2}$. For fixed $\delta \in(0,1]$ consider the solution $\psi_{\delta}$ of

$$
\begin{gathered}
\psi^{\prime \prime}+\left(M_{2}+1\right) \psi^{-e_{n}}=0 \text { in }(-L, L), \\
\psi(-L)=\psi(L)=\delta>0,
\end{gathered}
$$

with $2 L=\operatorname{diam} \Omega$. The function $\psi_{\delta}$ and the constant $\delta$ will serve as a pair of strict upper and lower solutions for an intermediate problem with the cut-off nonlinearity $f_{\delta}^{(j)}(x, u)$ defined by

$$
f_{\delta}^{(j)}(x, u)= \begin{cases}(\delta / 2)^{e_{j}} & \text { if } 0<u \leq \delta / 2 \\ u^{e_{j}} & \text { if } \delta \leq u \leq \psi_{\delta}(x), \\ \left(\psi_{\delta}(x)+1\right)^{e_{j}} & \text { if } \psi_{\delta}(x)+1 \leq u\end{cases}
$$

and extended in a nondecreasing, smooth way in the strips $\delta / 2 \leq u \leq \delta$ and $\psi_{\delta}(x) \leq u \leq \psi_{\delta}(x)+1$. With this construction $f_{\delta}^{(j)}$ is a bounded, nondecreasing, $\mathcal{C}^{1}$-function that coincides with $u^{e_{j}}$ in the region where $\delta \leq u \leq \psi_{\delta}(x)$. If $u_{\delta}$ is the solution of

$$
\begin{aligned}
\mathcal{F}_{\delta}(u):=\sum_{j=1}^{n} f_{\delta}^{(j)}(x, u) u_{x_{j} x_{j}}+q(x) & =0 \text { in } \Omega, \\
u & =\delta \text { on } \partial \Omega,
\end{aligned}
$$

then, due to $\mathcal{F}_{\delta}\left(\psi_{\delta}\right)<0, \mathcal{F}_{\delta}(\delta)>0$ we find by Lemma 4.1 that $\delta \leq u_{\delta} \leq \psi_{\delta}$. Thus we can remove the cut-off and find that $u_{\delta}$ solves

$$
\mathcal{F}(u)=0 \text { in } \Omega, \quad u=\delta \text { on } \partial \Omega .
$$

To find uniform lower bounds on $u_{\delta}$ in balls $B_{R}(\tilde{x}) \subset \Omega$ we consider the solution $\Phi:[0, R) \rightarrow \mathbb{R}^{+}$of

$$
\begin{gathered}
\Phi^{\prime \prime}+\frac{M_{1}}{n} \Phi^{-e_{n}}=0 \text { in }[0, R), \\
\Phi(R)=0, \quad \Phi^{\prime}(0)=0,
\end{gathered}
$$

where $R$ is chosen so small such that $\Phi \leq 1$ on $[0, R]$. Similarly to the argument in Section 3 the fact that $\Phi$ is decreasing and concave implies that the function $\phi(x)=\Phi(r)$ with $r=|x-\tilde{x}|$ defined on the ball $B_{R}(\tilde{x})$ satisfies $\Delta \phi+\phi^{-e_{n}} q(x) \geq 0$ and $\phi_{x_{j} x_{j}} \leq 0$. This implies

$$
\sum_{i=1}^{n} \phi^{e_{i}} \phi_{x_{i} x_{i}} \geq \phi^{e_{n}} \sum_{i=1}^{n} \phi_{x_{i} x_{i}} \geq-q(x)
$$

in $B_{R}(\tilde{x})$. Hence $\phi$ is a lower solution and we can apply Lemma 4.1 to $u_{\delta}$ and $\phi$ on the ball $B_{R}(\tilde{x})$ and find $u_{\delta} \geq \phi$ in $B_{R}(\tilde{x})$. By the standard covering argument 
we find that for $\delta \in(0,1]$ the family of functions $u_{\delta}$ is uniformly bounded away from zero on every compact subset $\Omega^{\prime}$ of $\Omega$. Applying the Krylov, Safonov and Schauder estimates, we find that $u_{\delta}$ is uniformly bounded in $\mathcal{C}^{2, \alpha}\left(\Omega^{\prime}\right)$. By extracting subsequences and diagonalizing, we find a sequence $u_{\delta}$ which converges for $\delta \rightarrow 0$ in $\mathcal{C}_{\text {loc }}^{2}(\Omega)$ to a limit function $u \in \mathcal{C}^{2}(\Omega)$ which satisfies $\mathcal{F}(u)=0$ in $\Omega$. Using Lemma 4.2 we find that $u_{\delta}$ is increasing in $\delta$. Hence

$$
\delta=\lim _{x \rightarrow \partial \Omega} u_{\delta}(x) \geq \lim _{x \rightarrow \partial \Omega} u(x) \geq 0
$$

for every $\delta \in(0,1]$, which shows that $u \in \mathcal{C}(\bar{\Omega})$ is the solution of (21). Uniqueness follows from Lemma 4.2 .

\section{Open questions}

Beyond the result that we have obtained here, the following questions remain open:

- Can one estimate the rate of growth of our solution near the boundary?

This question was considered by Loewner and Nirenberg [11, Bandle and Marcus [1], and Lazer and McKenna 9].

- As a first step towards the solution of the previous question, one may want to investigate the solutions of (2) and (3) on a half-space $x_{i_{0}}>0$ for some $i_{0}$. The conjecture is that the solutions only depend on $x_{i_{0}}$ and are hence solutions of a corresponding ordinary differential equation.

- Can one prove uniqueness on more general domains and without monotonicity assumptions on the functions $p, q$ ?

- Can one prove existence for more general singular quasilinear elliptic operators?

\section{ACKNOWLEDGMENT}

The third author greatfully acknowledges the support of his research by the Deutsche Forschungsgemeinschaft. The present paper was initiated during his visit at the University of Connecticut in September 1997. He thanks P.J. McKenna for the invitation and inspiring discussions.

\section{REFERENCES}

[1] C. Bandle and M. Marcus Large solutions of semilinear elliptic equations: existence, uniqueness and asymptotic behavior. J. d'Analyse Math., 58:9-24, 1992. MR 94c:35081

[2] C. Bandle and M. Marcus Asymptotic behaviour of solutions and their derivatives, for semilinear elliptic problems with blowup on the boundary. Ann. Inst. H. Poincaré Anal. Non Linéaire, 12:155-171, 1995. MR 96e:35038

[3] Y.S. Choi, A.C. Lazer and P.J. McKenna On a Singular Quasilinear Anisotropic Elliptic Boundary Value Problem. Trans. Amer. Math. Soc., 347:2633-2641, 1995. MR 95i:35087]

[4] Y.S. Choi and P.J. McKenna A singular quasilinear anisotropic elliptic boundary value problem. II. Trans. Amer. Math. Soc., 350:2925-2937, 1998. MR 98k:35039

[5] D. Gilbarg and N. Trudinger Elliptic Partial Differential Equations of Second Order, Second Edition. Springer-Verlag, New York, 1983. MR 86c:35035

[6] S. Hill and K.S. Moore An Existence Theorem for a Singular Quasilinear Elliptic Boundary Value Problem with Boundary Blowup. Nonlinear Analysis. To appear.

[7] J.B. Keller On solutions of $\triangle u=f(u)$. Comm. Pure Appl. Math., 10:503-510, 1957. MR 19:964c

[8] A. Lair and A. Shaker Uniqueness of solution to a singular quasilinear elliptic problem. Nonlinear Anal., 28:489-493, 1997. MR 98a:35039 
[9] A.C. Lazer and P.J. McKenna Asymptotic behavior of solutions of boundary blow-up problems. Differential and Integral Equations, 7:1001-1019, 1994. MR 95c:35084

[10] A.C. Lazer and P.J. McKenna On singular boundary value problems for the Monge-Ampère operator. J. Math. Anal. Appl., 197:341-362, 1996. [MR 97c:35064]

[11] C. Loewner and L. Nirenberg Partial differential equations invariant under conformal or projective transformations. Contributions to Analysis (L. Ahlfors ed.), Acad. Press N.Y., 1974, pp. 245-272. MR 50:10543

[12] R. Osserman On the inequality $\Delta u \geq f(u)$. Pacific J. Math., 7:1641-1647, 1957. MR 20:4701

[13] W. Reichel Uniqueness for degenerate elliptic equations via Serrin's sweeping principle. Intern. Series of Numer. Math., 123:375-387, 1997. MR 98h:35086

Department of Mathematics, Rowan University, Glassboro, New Jersey 08028

Department of Mathematics, University of Connecticut, Storrs, Connecticut 06269 Current address, K. S. Moore: Department of Mathematics, University of Michigan, 525 East University Ave., Ann Arbor, Michigan 48109-1109

E-mail address: ksmoore@math.lsa.umich.edu

Mathematisches Institut, Universität Zu Köln, 50931 Köln, Germany

Current address, W. Reichel: Mathematisches Institut, Universität Basel, Rheinsprung 21, CH-4051 Basel, Switzerland

E-mail address: reichel@eichler.math-lab.unibas.ch 